\title{
A note on generalized convex functions
}

Syed Zaheer Ullah', Muhammad Adil Khan ${ }^{1}$ and Yu-Ming Chu ${ }^{2,33^{*}}$

*Correspondence:

chuyuming2005@126.com

2Department of Mathematics,

Huzhou University, Huzhou, China

${ }^{3}$ School of Mathematics and

Statistics, Changsha University of

Science and Technology, Changsha,

China

Full list of author information is

available at the end of the article

\begin{abstract}
In the article, we provide an example for a $\eta$-convex function defined on rectangle is not convex, prove that every $\eta$-convex function defined on rectangle is coordinate $\eta$-convex and its converse is not true in general, define the coordinate $\left(\eta_{1}, \eta_{2}\right)$-convex function and establish its Hermite-Hadamard type inequality.
\end{abstract}

MSC: 26D15; 26A51;39B62

Keywords: Convex function; Coordinate convex function; $\eta$-convex function; Coordinate $\left(\eta_{1}, \eta_{2}\right)$-convex function

\section{Introduction}

Let $I \subseteq \mathbb{R}$ be an interval. Then a real-valued function $\Psi: I \mapsto \mathbb{R}$ is said to be convex on $I$ if the inequality

$$
\Psi[\lambda a+(1-\lambda) b] \leq \lambda \Psi(a)+(1-\lambda) \Psi(b)
$$

holds for all $a, b \in I$ and $\lambda \in(0,1) . \Psi$ is said to be concave if inequality (1.1) is reversed.

It is well known that the convexity theory has wide applications in special functions [1-30], differential equations [31-61] and bivariate means [62-67]. Recently, the extensions, generalizations, refinements and variants for the convexity have attracted the attention of many researchers. For example, Schur convexity [68-70], GA-convexity [71], GG-convexity [72], $s$-convexity [73, 74], preinvexity [75], strong convexity [76-79] and others [80-85].

Dragomir [86] defined the coordinate convex as follows.

Definition 1.1 (See [86]) Let $I_{1}, I_{2} \subseteq \mathbb{R}$ be two interval, $\Psi: I_{1} \times I_{2} \mapsto \mathbb{R}$ be a real-valued function, and the partial mappings $\Psi_{y}: I_{1} \mapsto \mathbb{R}$ and $\Psi_{x}: I_{2} \mapsto \mathbb{R}$ be defined by

$$
\Psi_{y}(u)=\Psi(u, y), \quad \Psi_{x}(v)=\Psi(x, v),
$$

respectively. Then $\Psi$ is said to be coordinate convex on $I_{1} \times I_{2}$ if $\Psi_{y}$ is convex on $I_{1}$ for all $y \in I_{2}$ and $\Psi_{x}$ is convex on $I_{2}$ for all $x \in I_{1}$.

Remark 1.2 Dragomir [86] proved that every convex function is coordinate convex, but not vice versa.

Next, we recall the concept of $\eta$-convexity which can be found in the literature [87].

(c) The Author(s) 2019. This article is distributed under the terms of the Creative Commons Attribution 4.0 International License (http://creativecommons.org/licenses/by/4.0/), which permits unrestricted use, distribution, and reproduction in any medium, provided you give appropriate credit to the original author(s) and the source, provide a link to the Creative Commons license, and indicate if changes were made. 
Definition 1.3 (See [87]) Let $I \subseteq \mathbb{R}$ be an interval, and $\Psi: I \mapsto \mathbb{R}$ and $\eta: \mathbb{R} \times \mathbb{R} \mapsto \mathbb{R}$ be two real-valued functions. Then $\Psi$ is said to be $\eta$-convex if the inequality

$$
\Psi[\mu x+(1-\mu) y] \leq \Psi(y)+\mu \eta[\Psi(x), \Psi(y)]
$$

holds for all $x, y \in I$ and $\mu \in[0,1]$.

Note that the $\eta$-convexity reduces to the usual convexity if $\eta(x, y)=x-y$ in Definition 1.3. The main purpose of the article is to give a non-trivial example for a $\eta$-convex function defined on rectangle is not convex, prove that every $\eta$-convex function defined on rectangle is coordinate $\eta$-convex but not vice versa, define the coordinate $\left(\eta_{1}, \eta_{2}\right)$-convex function and establish its Hermite-Hadamard type inequality.

\section{Main results}

To begin this section, it is interesting to give the definition of $\eta$-convex function defined on rectangle, and give a non-trivial example for a $\eta$-convex function defined on rectangle is not convex.

Definition 2.1 Let $I_{1}, I_{2} \subseteq \mathbb{R}$ be two intervals, and $\Psi: I_{1} \times I_{2} \mapsto \mathbb{R}$ and $\eta: \mathbb{R} \times \mathbb{R} \mapsto \mathbb{R}$ be two real-valued functions. Then $\Psi$ is said to be $\eta$-convex if the inequality

$$
\Psi[\mu x+(1-\mu) z, \mu y+(1-\mu) w] \leq \Psi(z, w)+\mu \eta[\Psi(x, y), \Psi(z, w)]
$$

holds for all $(x, y),(z, w) \in I_{1} \times I_{2}$ and $\mu \in[0,1]$.

Example 2.2 Let $\Psi:[1,5] \times[1,5] \mapsto \mathbb{R}$ and $\eta: \mathbb{R} \times \mathbb{R} \mapsto \mathbb{R}$ be defined by

$$
\Psi(x, y)=x^{2} y^{2}, \quad \eta(x, y)=104 x+103 y .
$$

Then $\Psi$ is $\eta$-convex on $[1,5] \times[1,5]$, but it is not convex.

Proof Let $\mu \in[0,1]$. Then for any $(x, y),(z, w) \in[1,5]$ we have

$$
\begin{aligned}
\Psi & {[\mu x+(1-\mu) z, \mu y+(1-\mu) w] } \\
= & {[\mu x+(1-\mu) z]^{2}[\mu y+(1-\mu) w]^{2} } \\
= & {\left[z^{2}+\mu\left(\mu x^{2}+\mu z^{2}-2 z^{2}\right)+2 \mu(1-\mu) x z\right] } \\
& \times\left[w^{2}+\mu\left(\mu y^{2}+\mu w^{2}-2 w^{2}\right)+2 \mu(1-\mu) y w\right] \\
\leq & {\left[z^{2}+\mu x^{2}+2 \mu(1-\mu) x z\right]\left[w^{2}+\mu y^{2}+2 \mu(1-\mu) y w\right] } \\
\leq & {\left[z^{2}+\mu x^{2}+\mu(1-\mu)\left(x^{2}+z^{2}\right)\right]\left[w^{2}+\mu y^{2}+\mu(1-\mu)\left(y^{2}+w^{2}\right)\right] } \\
\leq & {\left[z^{2}+\mu\left(x^{2}+x^{2}+z^{2}\right)\right]\left[w^{2}+\mu\left(y^{2}+y^{2}+w^{2}\right)\right] } \\
= & z^{2} w^{2}+\mu\left[2 y^{2} z^{2}+z^{2} w^{2}+2 x^{2} w^{2}+w^{2} z^{2}\right]+\mu^{2}\left[4 x^{2} y^{2}+2 x^{2} w^{2}+2 y^{2} z^{2}+z^{2} w^{2}\right] \\
\leq & \Psi(z, w)+\mu\left[2 y^{2} z^{2}+z^{2} w^{2}+2 x^{2} w^{2}+w^{2} z^{2}\right]+\mu\left[4 x^{2} y^{2}+2 x^{2} w^{2}+2 y^{2} z^{2}+z^{2} w^{2}\right] \\
= & \Psi(z, w)+\mu\left[4 x^{2} y^{2}+3 z^{2} w^{2}+4\left(z^{2} y^{2}+x^{2} w^{2}\right)\right] .
\end{aligned}
$$


Note that

$$
z^{2} \leq 25 x^{2}, \quad x^{2} \leq 25 z^{2}
$$

It follows from (2.1) and (2.2) that

$$
\begin{aligned}
& \Psi[\mu x+(1-\mu) z, \mu y+(1-\mu) w] \\
& \quad \leq \Psi(z, w)+\mu\left[104 x^{2} y^{2}+103 z^{2} w^{2}\right] \\
& =\Psi(z, w)+\mu \eta[\Psi(x, y), \Psi(z, w)],
\end{aligned}
$$

which shows that $\Psi$ is $\eta$-convex on $[1,5] \times[1,5]$. It is easily to verify that $\Psi$ is not convex on $[1,5] \times[1,5]$, for details see $[79]$.

Next, we introduce the definition of coordinate $\left(\eta_{1}, \eta_{2}\right)$-convexity.

Definition 2.3 Let $I_{1}, I_{2} \subseteq \mathbb{R}$ be two intervals, $\Psi: I_{1} \times I_{2} \mapsto \mathbb{R}, \eta_{1}, \eta_{2}: \mathbb{R} \times \mathbb{R} \mapsto \mathbb{R}$ be three real-valued functions, and the partial mappings $\Psi_{y}: I_{1} \mapsto \mathbb{R}$ and $\Psi_{x}: I_{2} \mapsto \mathbb{R}$ be defined by

$$
\Psi_{y}(u)=\Psi(u, y), \quad \Psi_{x}(v)=\Psi(x, v) .
$$

Then $\Psi$ is said to be coordinate $\left(\eta_{1}, \eta_{2}\right)$-convex on $I_{1} \times I_{2}$ if $\Psi_{y}$ is $\eta_{1}$-convex on $I_{1}$ and $\Psi_{x}$ is $\eta_{2}$-convex on $I_{2}$. In particular, if $\eta_{1}=\eta_{2}=\eta$, then $\Psi$ is said to be coordinate $\eta$-convex.

Example 2.4 Let $\Psi:[0, \infty) \times[0, \infty) \mapsto \mathbb{R}$ be defined by $\Psi(x, y)=-|x|-y^{2}, \eta_{1}(x, y)=-x-y$ and $\eta_{2}(x, y)=-x-2 y$. Then $\Psi$ is coordinate $\left(\eta_{1}, \eta_{2}\right)$-convex on $[0, \infty) \times[0, \infty)$.

Proof Let $x_{1}, y_{1} \in[0, \infty)$ and $\mu \in[0,1]$. Then for any $(x, y) \in[0, \infty)$ we clearly see that

$$
\begin{aligned}
& \Psi_{y}\left(\mu x_{1}+(1-\mu) x_{2}\right)=-\left|\mu x_{1}+(1-\mu) x_{2}\right|-y^{2}, \\
& \Psi_{y}\left(x_{2}\right)+\mu \eta_{1}\left(\Psi_{y}\left(x_{1}\right), \Psi_{y}\left(x_{2}\right)\right) \\
& \quad=-\left|x_{2}\right|-y^{2}+\mu \eta_{1}\left(-\left|x_{1}\right|-y^{2},-\left|x_{2}\right|-y^{2}\right) \\
& \quad=-\left|x_{2}\right|-y^{2}+\mu\left(\left|x_{1}\right|+\left|x_{2}\right|+2 y^{2}\right), \\
& \Psi_{x}\left(\mu y_{1}+(1-\mu) y_{2}\right)=-|x|-\left(\mu y_{1}+(1-\mu) y_{2}\right)^{2}, \\
& \Psi_{x}\left(y_{2}\right)+\mu \eta_{2}\left(\Psi_{x}\left(y_{1}\right), \Psi_{x}\left(y_{2}\right)\right) \\
& \quad=-|x|-y_{2}^{2}+\mu \eta_{2}\left(-|x|-y_{1}^{2},-|x|-y_{2}^{2}\right) \\
& \quad=-|x|-y_{2}^{2}+\mu\left(y_{1}^{2}+2 y_{2}^{2}+3|x|\right) .
\end{aligned}
$$

It follows from (2.3)-(2.6) that

$$
\begin{aligned}
& \Psi_{y}\left(x_{2}\right)+\mu \eta_{1}\left(\Psi_{y}\left(x_{1}\right), \Psi_{y}\left(x_{2}\right)\right)-\Psi_{y}\left(\mu x_{1}+(1-\mu) x_{2}\right) \\
& \quad=\mu\left(\left|x_{1}\right|+\left|x_{2}\right|+2 y^{2}\right)+\left|\mu x_{1}+(1-\mu) x_{2}\right|-\left|x_{2}\right| \\
& \quad \geq 2 \mu y^{2}+\mu\left|x_{1}\right|+\mu\left|x_{2}\right|+(1-\mu)\left|x_{2}\right|-\mu\left|x_{1}\right|-\left|x_{2}\right|
\end{aligned}
$$




$$
\begin{aligned}
& =2 \mu y^{2} \geq 0, \\
& \Psi_{x}\left(y_{2}\right)+\mu \eta_{2}\left(\Psi_{x}\left(y_{1}\right), \Psi_{x}\left(y_{2}\right)\right)-\Psi_{x}\left(\mu y_{1}+(1-\mu) y_{2}\right) \\
& =3 \mu|x|+2 \mu(1-\mu) y_{1} y_{2}+\mu(1+\mu) y_{1}^{2}+\mu^{2} y_{2}^{2} \geq 0 .
\end{aligned}
$$

Therefore, $\Psi$ is coordinate $\left(\eta_{1}, \eta_{2}\right)$-convex on $[0, \infty) \times[0, \infty)$ follows from $(2.7)$ and (2.8).

Theorem 2.5 Let $I_{1}, I_{2} \subseteq \mathbb{R}$ be two interval and $\eta: \mathbb{R} \times \mathbb{R} \mapsto \mathbb{R}$ be a real-valued function. Then $\Psi$ is coordinate $\eta$-convex on $I_{1} \times I_{2}$ if $\Psi$ is $\eta$-convex on $I_{1} \times I_{2}$.

Proof Let $(x, y) \in I_{1} \times I_{2}, u, v \in I_{2}$ and $z, w \in I_{1}$. Then it follows from the $\eta$-convexity of the function $\Psi$ on $I_{1} \times I_{2}$ that

$$
\begin{aligned}
\Psi_{x}(\mu \nu+(1-\mu) u) & =\Psi(x, \mu v+(1-\mu) u) \\
& =\Psi(\mu x+(1-\mu) x, \mu v+(1-\mu) u) \\
& \leq \Psi(x, u)+\mu \eta(\Psi(x, v), \Psi(x, u)) \\
& =\Psi_{x}(u)+\mu \eta\left(\Psi_{x}(v), \Psi_{x}(u)\right)
\end{aligned}
$$

and

$$
\begin{aligned}
\Psi_{y}(\mu z+(1-\mu) w) & =\Psi(\mu z+(1-\mu) w, y) \\
& =\Psi(\mu z+(1-\mu) w, \mu y+(1-\mu) y) \\
& \leq \Psi(w, y)+\mu \eta(\Psi(z, y), \Psi(w, y)) \\
& =\Psi_{y}(w)+\mu \eta\left(\Psi_{y}(z), \Psi_{y}(w)\right) .
\end{aligned}
$$

Inequalities (2.9) and (2.10) imply that $\Psi_{x}$ is $\eta$-convex on $I_{2}$ and $\Psi_{y}$ is $\eta$-convex on $I_{1}$. Therefore, $\Psi$ is coordinate $\eta$-convex on $I_{1} \times I_{2}$.

Example 2.6 Let $I_{1}=I_{2}=[0, \infty), \Psi, \eta: I_{1} \times I_{2} \mapsto[0, \infty)$ be defined by

$$
\Psi(x, y)=x y, \quad \eta(x, y)=x+y .
$$

Then $\Psi$ is coordinate $\eta$-convex on $I_{1} \times I_{2}$ but it is not $\eta$-convex on $I_{1} \times I_{2}$.

Proof Let $x, y, u, v, z, w \in[0, \infty)$ and $\mu \in[0,1]$. Then it follows from (2.11) that

$$
\begin{aligned}
& \begin{aligned}
\Psi_{x}(\mu u+(1-\mu) v) & =\Psi(x, \mu u+(1-\mu) v) \\
= & x(\mu u+(1-\mu) v)=-\mu x v+x(\mu u+v), \\
\Psi(x, v)+\mu \eta(\Psi(x, u), \Psi(x, v)) & =x v+\mu \eta(x u, x v) \\
& =x v+\mu(x u+x v)=\mu x v+x(\mu u+v),
\end{aligned} \\
& \Psi_{y}(\mu z+(1-\mu) w)=\Psi(\mu z+(1-\mu) w, y)
\end{aligned}
$$




$$
\begin{aligned}
=y(\mu z+(1-\mu) w) & =-\mu y w+y(\mu z+w) \\
\Psi(w, y)+\mu \eta(\Psi(z, y), \Psi(w, y)) & =w y+\mu \eta(z y, w y) \\
& =w y+\mu(z y+w y)=\mu y w+y(\mu z+w) .
\end{aligned}
$$

Inequalities (2.12)-(2.15) imply that

$$
\Psi_{x}(\mu u+(1-\mu) v) \leq \Psi(x, v)+\mu \eta(\Psi(x, u), \Psi(x, v))
$$

and

$$
\Psi_{y}(\mu z+(1-\mu) w) \leq \Psi(w, y)+\mu \eta(\Psi(z, y), \Psi(w, y))
$$

Note that

$$
\Psi_{x}(\mu u+(1-\mu) v)=\Psi(\mu x+(1-\mu) x, \mu u+(1-\mu) v)
$$

and

$$
\Psi_{y}(\mu z+(1-\mu) w)=\Psi(\mu z+(1-\mu) w, \mu y+(1-\mu) y) .
$$

Therefore, $\Psi$ is coordinate $\eta$-convex on $I_{1} \times I_{2}$ follows from (2.16)-(2.19).

Next, we prove that $\Psi$ is not $\eta$-convex on $I_{1} \times I_{2}$.

Let $\mu \in(0,1), x=w=1$ and $y=z=0$. Then (2.11) leads to

$$
\begin{aligned}
& \Psi(\mu x+(1-\mu) z, \mu y+(1-\mu) w) \\
& \quad=\Psi(\mu, 1-\mu)=\mu(1-\mu)>0, \\
& \Psi(z, w)+\mu \eta(\Psi(x, y), \Psi(z, w)) \\
& \quad=\Psi(0,1)+\mu \eta(\Psi(1,0), \Psi(0,1))=0 .
\end{aligned}
$$

From (2.20) and (2.21) we clearly see that $\Psi$ is not $\eta$-convex on $I_{1} \times I_{2}$.

Next, we establish a Hermite-Hadamard type inequality for the coordinate $\left(\eta_{1}, \eta_{2}\right)$ convex function.

Theorem 2.7 Let $a, b, c, d \in \mathbb{R}$ with $a<b$ and $c<d, \Psi:[a, b] \times[c, d] \mapsto \mathbb{R}, \eta_{1}, \eta_{2}: \mathbb{R} \times \mathbb{R} \mapsto$ $\mathbb{R}$ be three real-valued functions such that $\Psi$ is coordinate $\left(\eta_{1}, \eta_{2}\right)$-convex on $[a, b] \times[c, d]$ and

$$
\eta_{1}(x, y) \leq M_{\eta_{1}}, \quad \eta_{2}(x, y) \leq M_{\eta_{2}}
$$


for all $x, y \in \mathbb{R}$, where $M_{\eta_{1}}$ and $M_{\eta_{2}}$ are two positive constants. Then

$$
\begin{aligned}
\Psi( & \left.\frac{a+b}{2}, \frac{c+d}{2}\right)-\frac{M_{\eta_{1}}+M_{\eta_{2}}}{2} \\
\leq & \frac{1}{2}\left[\frac{1}{b-a} \int_{a}^{b} \Psi\left(x, \frac{c+d}{2}\right) d x+\frac{1}{d-c} \int_{c}^{d} \Psi\left(\frac{a+b}{2}, y\right) d y\right]-\frac{M_{\eta_{1}}+M_{\eta_{2}}}{4} \\
\leq & \frac{1}{(b-a)(d-c)} \int_{c}^{d} \int_{a}^{b} \Psi(x, y) d x d y \\
\leq & \frac{1}{4}\left[\frac{1}{b-a} \int_{a}^{b}(\Psi(x, c)+\Psi(x, d)) d x+\frac{1}{d-c} \int_{c}^{d}(\Psi(a, y)+\Psi(b, y)) d y\right] \\
& +\frac{M_{\eta_{1}}+M_{\eta_{2}}}{4} \\
\leq & \frac{1}{4}[\Psi(a, c)+\Psi(b, c)+\Psi(a, d)+\Psi(b, d)]+\frac{5}{4}\left[M_{\eta_{1}}+M_{\eta_{2}}\right] .
\end{aligned}
$$

Proof For any fixed $x \in[a, b], \Psi_{x}(y)=\Psi(x, y)$ is $\eta_{2}$-convex on $[c, d]$ due to $\Psi$ is coordinate $\left(\eta_{1}, \eta_{2}\right)$-convex on $[a, b] \times[c, d]$. It follows from [77, Theorem 5] that

$$
\Psi\left(x, \frac{c+d}{2}\right)-\frac{M_{\eta_{2}}}{2} \leq \frac{1}{d-c} \int_{c}^{d} \Psi(x, y) d y \leq \frac{\Psi(x, c)+\Psi(x, d)}{2}+\frac{M_{\eta_{2}}}{2} .
$$

Integrating each side of inequality (2.23) with respect to the variable $x$ on $[a, b]$ leads to

$$
\begin{aligned}
& \frac{1}{b-a} \int_{a}^{b} \Psi\left(x, \frac{c+d}{2}\right) d x-\frac{M_{\eta_{2}}}{2} \\
& \quad \leq \frac{1}{(b-a)(d-c)} \int_{c}^{d} \int_{a}^{b} \Psi(x, y) d x d y \\
& \leq \frac{1}{2(b-a)} \int_{a}^{b}[\Psi(x, c)+\Psi(x, d)] d x+\frac{M_{\eta_{2}}}{2} .
\end{aligned}
$$

By similar arguments we have

$$
\begin{aligned}
& \frac{1}{d-c} \int_{c}^{d} \Psi\left(\frac{a+b}{2}, y\right) d y-\frac{M_{\eta_{1}}}{2} \\
& \leq \frac{1}{(b-a)(d-c)} \int_{c}^{d} \int_{a}^{b} \Psi(x, y) d x d y \\
& \leq \frac{1}{2(d-c)} \int_{c}^{d}[\Psi(a, y)+\Psi(b, y)] d y+\frac{M_{\eta_{1}}}{2} .
\end{aligned}
$$

Adding (2.24) and (2.25) we get the second and third inequalities of (2.22).

Making use of the $\left(\eta_{1}, \eta_{2}\right)$-convexity of the function $\Psi$ on $[a, b] \times[c, d]$ and $[88$, Theorem 5] again we get

$$
\begin{aligned}
& \Psi\left(\frac{a+b}{2}, \frac{c+d}{2}\right)-\frac{M_{\eta_{2}}}{2} \leq \frac{1}{b-a} \int_{a}^{b} \Psi\left(x, \frac{c+d}{2}\right) d x \\
& \Psi\left(\frac{a+b}{2}, \frac{c+d}{2}\right)-\frac{M_{\eta_{1}}}{2} \leq \frac{1}{d-c} \int_{c}^{d} \Psi\left(\frac{a+b}{2}, y\right) d y
\end{aligned}
$$




$$
\begin{aligned}
& \frac{1}{b-a} \int_{a}^{b} \Psi(x, c) d x \leq \frac{\Psi(a, c)+\Psi(b, c)}{2}+\frac{M_{\eta_{2}}}{2}, \\
& \frac{1}{b-a} \int_{a}^{b} \Psi(x, d) d x \leq \frac{\Psi(a, d)+\Psi(b, d)}{2}+\frac{M_{\eta_{2}}}{2}, \\
& \frac{1}{d-c} \int_{c}^{d} \Psi(a, y) d y \leq \frac{\Psi(a, c)+\Psi(a, d)}{2}+\frac{M_{\eta_{1}}}{2}, \\
& \frac{1}{d-c} \int_{c}^{d} \Psi(b, y) d y \leq \frac{\Psi(b, c)+\Psi(b, d)}{2}+\frac{M_{\eta_{1}}}{2} .
\end{aligned}
$$

Therefore, the first inequality of (2.22) follows from (2.26) and (2.27) with adding $-\frac{1}{2} M_{\eta_{2}}$ and $-\frac{1}{2} M_{\eta_{1}}$ respectively, and the last inequality in (2.22) can be derived from (2.28)-(2.31) immediately, with adding $\frac{1}{4}\left[M_{\eta_{1}}+M_{\eta_{2}}\right]$.

\section{Results and discussion}

In the article, we establish a non-trivial example for a $\eta$-convex function defined on rectangle is not convex, prove that every $\eta$-convex function defined on rectangle is coordinate $\eta$-convex and its converse is not true in general. Furthermore, we define a new class of function which is coordinate $\left(\eta_{1}, \eta_{2}\right)$-convex function and prove its well-known HermiteHadamard type inequality.

\section{Conclusion}

We find an example for $\eta$-convex function defined on rectangle is not convex. The authors define a coordinate $\left(\eta_{1}, \eta_{2}\right)$-convex function and prove its results. Our approach may have further applications in the theory of $\eta$-convexity.

Funding

This work was supported by the Natural Science Foundation of China (Grant Nos. 61673169, 11301127, 11701176,

$11626101,11601485)$

Availability of data and materials

Not applicable.

Competing interests

The authors declare that they have no competing interests.

Authors' contributions

All authors contributed equally to the writing of this paper. All authors read and approved the final manuscript.

Author details

'Department of Mathematics, University of Peshawar, Peshawar, Pakistan. ${ }^{2}$ Department of Mathematics, Huzhou University, Huzhou, China. ${ }^{3}$ School of Mathematics and Statistics, Changsha University of Science and Technology, Changsha, China.

\section{Publisher's Note}

Springer Nature remains neutral with regard to jurisdictional claims in published maps and institutional affiliations.

Received: 11 July 2019 Accepted: 29 October 2019 Published online: 12 November 2019

\section{References}

1. Guessab, A., Schmeisser, G.: Sharp integral inequalities of the Hermite-Hadamard type. J. Approx. Theory 115(2), 260-288 (2002)

2. Shi, H.-P., Zhang, H.-Q.: Existence of gap solitons in periodic discrete nonlinear Schrödinger equations. J. Math. Anal. Appl. 361(2), 411-419 (2010)

3. Zhou, W.-J., Zhang, L.: Convergence of a regularized factorized quasi-Newton method for nonlinear least squares problems. Comput. Appl. Math. 29(2), 195-214 (2010)

4. Yang, X.-S., Zhu, Q.-X., Huang, C.-X.: Generalized lag-synchronization of chaotic mix-delayed systems with uncertain parameters and unknown perturbations. Nonlinear Anal., Real World Appl. 12(1), 93-105 (2011) 
5. Zhu, Q.-X., Huang, C.-X., Yang, X.-S.: Exponential stability for stochastic jumping BAM neural networks with time-varying and distributed delays. Nonlinear Anal. Hybrid Syst. 5(1), 52-77 (2011)

6. Dai, Z.-F., Wen, F.-H.: A modified CG-DESCENT method for unconstrained optimization. J. Comput. Appl. Math 235(11), 3332-3341 (2011)

7. Gou, K., Sun, B.: Numerical solution of the Goursat problem on a triangular domain with mixed boundary conditions. Appl. Math. Comput. 217(21), 8765-8777 (2011)

8. Lin, L., Liu, Z.-Y.: An alternating projected gradient algorithm for nonnegative matrix factorization. Appl. Math. Comput. 217(24), 9997-10002 (2011)

9. Zhang, L., Li, J.-L.: A new globalization technique for nonlinear conjugate gradient methods for nonconvex minimization. Appl. Math. Comput. 217(24), 10295-10304 (2011)

10. Xiao, C.-E., Liu, J.-B., Liu, Y.-L.: An inverse pollution problem in porous media. Appl. Math. Comput. 218(7), 3649-3653 (2011)

11. Huang, C.-X., Liu, L.-Z: Sharp function inequalities and boundness for Toeplitz type operator related to general fractional singular integral operator. Publ. Inst. Math. 92(106), 165-176 (2012)

12. Zhou, W.-.:: On the convergence of the modified Levenberg-Marquardt method with a nonmonotone second order Armijo type line search. J. Comput. Appl. Math. 239, 152-161 (2013)

13. Zhang, L., Jian, S.-Y.: Further studies on the Wei-Yao-Liu nonlinear conjugate gradient method. Appl. Math. Comput. 219(14), 7616-7621 (2013)

14. Li, X.-F., Tang, G.-J., Tang, B.-Q.: Stress field around a strike-slip fault in orthotropic elastic layers via a hypersingular integral equation. Comput. Math. Appl. 66(11), 2317-2326 (2013)

15. Qin, G.-X., Huang, C.-X., Xie, Y.-Q., Wen, F.-H.: Asymptotic behavior for third-order quasi-linear differential equations. Adv. Differ. Equ. 2013, Article ID 305 (2013)

16. Zhou, W.-J., Chen, X.-L.: On the convergence of a modified regularized Newton method for convex optimization with singular solutions. J. Comput. Appl. Math. 239, 179-188 (2013)

17. Wang, M.-K., Chu, Y.-M.: Asymptotical bounds for complete elliptic integrals of the second kind. J. Math. Anal. Appl. 402(1), 119-126 (2013)

18. Wang, G.-D., Zhang, X.-H., Chu, Y.-M.: A power mean inequality involving the complete elliptic integrals. Rocky Mt. J. Math. 44(5), 1661-1667 (2014)

19. Wang, M.-K., Chu, Y.-M., Jiang, Y.-P.: Ramanujan's cubic transformation inequalities for zero-balanced hypergeometric functions. Rocky Mt. J. Math. 46(2), 679-691 (2016)

20. Yang, Z.-H., Qian, W.-M., Chu, Y.-M., Zhang, W.: Monotonicity rule for the quotient of two functions and its application. J. Inequal. Appl. 2017, Article ID 106 (2017)

21. Yang, Z.-H., Qian, W.-M., Chu, Y.-M., Zhang, W.: On rational bounds for the gamma function. J. Inequal. Appl. 2017, Article ID 210 (2017)

22. Huang, T.-R., Han, B.-W., Ma, X.-Y., Chu, Y.-M.: Optimal bounds for the generalized Euler-Mascheroni constant. J. Inequal. Appl. 2018, Article ID 118 (2018)

23. Huang, T.-R., Tan, S.-Y., Ma, X.-Y., Chu, Y.-M.: Monotonicity properties and bounds for the complete $p$-elliptic integrals. J. Inequal. Appl. 2018, Article ID 239 (2018)

24. Yang, Z.-H., Qian, W.-M., Chu, Y.-M.: Monotonicity properties and bounds involving the complete elliptic integrals of the first kind. Math. Inequal. Appl. 21(4), 1185-1199 (2018)

25. Yang, Z.-H., Chu, Y.-M., Zhang, W.: High accuracy asymptotic bounds for the complete elliptic integral of the second kind. Appl. Math. Comput. 348, 552-564 (2019)

26. Qiu, S.-L., Ma, X.Y., Chu, Y.-M.: Sharp Landen transformation inequalities for hypergeometric functions, with applications. J. Math. Anal. Appl. 474(2), 1306-1337 (2019)

27. Wang, M.-K., Chu, Y.-M., Zhang, W.: Monotonicity and inequalities involving zero-balanced hypergeometric function. Math. Inequal. Appl. 22(2), 601-617 (2019)

28. Wang, M.-K., Chu, Y.-M., Zhang, W.: Precise estimates for the solution of Ramanujan's generalized modular equation. Ramanujan J. 49(3), 653-668 (2019)

29. Wang, M.-K., Zhang, W., Chu, Y.-M.: Monotonicity, convexity and inequalities involving the generalized elliptic integrals. Acta Math. Sci. 39B(5), 1440-1450 (2019)

30. Wang, M.-K., Chu, H.-H., Chu, Y.-M.: Precise bounds for the weighted Hölder mean of the complete $p$-elliptic integrals. J. Math. Anal. Appl. https://doi.org/10.1016/j.jmaa.2019.123388

31. Huang, C.-X., Yang, Z.-C., Yi, T.-S., Zou, X.-F.: On the basins of attraction for a class of delay differential equations with non-monotone bistable nonlinearities. J. Differ. Equ. 256(7), 2101-2114 (2014)

32. Tang, W.-S., Sun, Y.-J.: Construction of Runge-Kutta type methods for solving ordinary differential equations. Appl. Math. Comput. 234, 179-191 (2014)

33. Huang, C.-X., Guo, S., Liu, L.-Z.: Boundedness on Morrey space for Toeplitz type operator associated to singular integral operator with variable Calderón-Zygmund kernel. J. Math. Inequal. 8(3), 453-464 (2014)

34. Xie, D.-X., Li, J.: A new analysis of electrostatic free energy minimization and Poisson-Boltzmann equation for protein in ionic solvent. Nonlinear Anal., Real World Appl. 21, 185-196 (2015)

35. Zhou, W.-J., Wang, F.: A PRP-based residual method for large-scale monotone nonlinear equations. Appl. Math. Comput. 261, 1-7 (2015)

36. Dai, Z.-F., Chen, X.-H., Wen, F.-H.: A modified Perry's conjugate gradient method-based derivative-free method for solving large-scale nonlinear monotone equations. Appl. Math. Comput. 270, 378-386 (2015)

37. Liu, Y.-C., Wu, J.: Multiple solutions of ordinary differential systems with min-max terms and applications to the fuzzy differential equations. Adv. Differ. Equ. 2015, Article ID 379 (2015)

38. Fang, X.-P., Deng, Y.-J., Li, J.: Plasmon resonance and heat generation in nanostructures. Math. Methods Appl. Sci. 38(18), 4663-4672 (2015)

39. Dai, Z.-F.: Comments on a new class of nonlinear conjugate gradient coefficients with global convergence properties. Appl. Math. Comput. 276, 297-300 (2016)

40. Li, J.-L., Sun, G.-Y., Zhang, R.-M.: The numerical solution of scattering by infinite rough interfaces based on the integral equation method. Comput. Math. Appl. 71(7), 1491-1502 (2016) 
41. Tan, Y.-X., Jing, K.: Existence and global exponential stability of almost periodic solution for delayed competitive neural networks with discontinuous activations. Math. Methods Appl. Sci. 39(11), 2821-2839 (2016)

42. Duan, L., Huang, C.-X.: Existence and global attractivity of almost periodic solutions for a delayed differential neoclassical growth model. Math. Methods Appl. Sci. 40(3), 814-822 (2017)

43. Duan, L., Huang, L.-H., Guo, Z.-Y., Fang, X.-W.: Periodic attractor for reaction-diffusion high-order Hopfield neural networks with time-varying delays. Comput. Math. Appl. 73(2), 233-245 (2017)

44. Wang, W.-S., Chen, Y.-Z.: Fast numerical valuation of options with jump under Merton's model. J. Comput. Appl. Math. 318, 79-92 (2017)

45. Huang, C.-X., Liu, L.-Z.: Boundedness of multilinear singular integral operator with a non-smooth kernel and mean oscillation. Quaest. Math. 40(3), 295-312 (2017)

46. Hu, H.-J., Liu, L.-Z.: Weighted inequalities for a general commutator associated to a singular integral operator satisfying a variant of Hörmander's condition. Math. Notes 101(5-6), 830-840 (2017)

47. Cai, Z.-W., Huang, J.-H., Huang, L.-H.: Generalized Lyapunov-Razumikhin method for retarded differential inclusions: applications to discontinuous neural networks. Discrete Contin. Dyn. Syst. 22B(9), 3591-3614 (2017)

48. Wang, W.-S.: On A-stable one-leg methods for solving nonlinear Volterra functional differential equations. Appl. Math. Comput. 314, 380-390 (2017)

49. Hu, H.-J., Zou, X.-F.: Existence of an extinction wave in the Fisher equation with a shifting habitat. Proc. Am. Math. Soc. 145(11), 4763-4771 (2017)

50. Tan, Y.-X., Huang, C.-X., Sun, B., Wang, T.: Dynamics of a class of delayed reaction-diffusion systems with Neumann boundary condition. J. Math. Anal. Appl. 458(2), 1115-1130 (2018)

51. Tang, W.-S., Zhang, J.-J.: Symplecticity-preserving continuous-stage Runge-Kutta-Nyström methods. Appl. Math. Comput. 323, 204-219 (2018)

52. Duan, L., Fang, X.-W., Huang, C.-X.: Global exponential convergence in a delayed almost periodic Nicholson's blowflies model with discontinuous harvesting. Math. Methods Appl. Sci. 41(5), 1954-1965 (2018)

53. Liu, Z.-Y., Wu, N.-C., Qin, X.-R., Zhang, Y.-L.: Trigonometric transform splitting methods for real symmetric Toeplitz systems. Comput. Math. Appl. 75(8), 2782-2794 (2018)

54. Huang, C.-X., Qiao, Y.-C., Huang, L.-H., Agarwal, R.P.: Dynamical behaviors of a food-chain model with stage structure and time delays. Adv. Differ. Equ. 2018, Article ID 186 (2018)

55. Cai, Z.-W., Huang, J.-H., Huang, L.-H.: Periodic orbit analysis for the delayed Filippov system. Proc. Am. Math. Soc 146(11), 4667-4682 (2018)

56. Wang, J.-F., Chen, X.-Y., Huang, L.-H.: The number and stability of limit cycles for planar piecewise linear systems of node-saddle type. J. Math. Anal. Appl. 469(1), 405-427 (2019)

57. Wang, J.-F., Huang, C.-X., Huang, L.-H.: Discontinuity-induced limit cycles in a general planar piecewise linear system of saddle-focus type. Nonlinear Anal. Hybrid Syst. 33, 162-178 (2019)

58. Jiang, Y.-J., Xu, X.-J.: A monotone finite volume method for time fractional Fokker-Planck equations. Sci. China Math 62(4), 783-794 (2019)

59. Peng, J., Zhang, Y.: Heron triangles with figurate number sides. Acta Math. Hung. 157(2), 478-488 (2019)

60. Tian, Z.-L., Liu, Y., Zhang, Y., Liu, Z.-Y., Tian, M.-Y.: The general inner-outer iteration method based on regular splittings for the PageRank problem. Appl. Math. Comput. 356, 479-501 (2019)

61. Wang, W.-S., Chen, Y.-Z., Fang, H.: On the variable two-step IMEX BDF method for parabolic integro-differential equations with nonsmooth initial data arising in finance. SIAM J. Numer. Anal. 57(3), 1289-1317 (2019)

62. Chu, Y.-M., Wang, M.-K., Qiu, S.-L.: Optimal combinations bounds of root-square and arithmetic means for Toader mean. Proc. Indian Acad. Sci. Math. Sci. 122(1), 41-51 (2012)

63. Zhao, T.-H., Zhou, B.-C., Wang, M.-K., Chu, Y.-M.: On approximating the quasi-arithmetic mean. J. Inequal. Appl. 2019, Article ID 42 (2019)

64. Wang, J.-L., Qian, W.-M., He, Z.-Y., Chu, Y.-M.: On approximating the Toader mean by other bivariate means. J. Funct. Spaces 2019, Article ID 6082413 (2019)

65. Qian, W.-M., Xu, H.-Z., Chu, Y.-M.: Improvements of bounds for the Sándor-Yang means. J. Inequal. Appl. 2019, Article ID 73 (2019)

66. He, X.-H., Qian, W.-M., Xu, H.-Z., Chu, Y.-M.: Sharp power mean bounds for two Sándor-Yang means. Rev. R. Acad. Cienc. Exactas Fís. Nat., Ser. A Mat. 113(3), 2627-2638 (2019)

67. Qian, W.-M., He, Z.-Y., Zhang, H.-W., Chu, Y.-M.: Sharp bounds for Neuman means in terms of two-parameter contraharmonic and arithmetic mean. J. Inequal. Appl. 2019, Article ID 168 (2019)

68. Chu, Y.-M., Wang, G.-D., Zhang, X.-H.: The Schur multiplicative and harmonic convexities of the complete symmetric function. Math. Nachr. 284(5-6), 653-663 (2011)

69. Chu, Y.-M., Xia, W.-F., Zhang, X.-H.: The Schur concavity, Schur multiplicative and harmonic convexities of the second dual form of the Hamy symmetric function with applications. J. Multivar. Anal. 105, 412-421 (2012)

70. $\mathrm{Wu}, \mathrm{S} .-\mathrm{H}_{\text {., }}$ Chu, Y.-M.: Schur $m$-power convexity of generalized geometric Bonferroni mean involving three parameters. J. Inequal. Appl. 2019, Article ID 57 (2019)

71. Zhang, X.-M., Chu, Y.-M., Zhang, X.-H.: The Hermite-Hadamard type inequality of GA-convex functions and its applications. J. Inequal. Appl. 2010, Article ID 507560 (2010)

72. Khurshid, Y., Adil Khan, M., Chu, Y.-M.: Conformable integral inequalities of the Hermite-Hadamard type in terms of GG- and GA-convexities. J. Funct. Spaces 2019, Article ID 6926107 (2019)

73. Adil Khan, M., Chu, Y.-M., Khan, T.U., Khan, J.: Some new inequalities of Hermite-Hadamard type for s-convex functions with applications. Open Math. 15(1), 1414-1430 (2017)

74. Adil Khan, M., Hanif, M., Khan, Z.A., Ahmad, K., Chu, Y.-M.: Association of Jensen's inequality for s-convex function with Csiszár divergence. J. Inequal. Appl. 2019, Article ID 162 (2019)

75. Khurshid, Y., Adil Khan, M., Chu, Y.-M., Khan, Z.A.: Hermite-Hadamard-Fejér inequalities for conformable fractional integrals via preinvex functions. J. Funct. Spaces 2019, Article ID 3146210 (2019)

76. Song, Y.-Q., Adil Khan, M., Zaheer Ullah, S., Chu, Y.-M.: Integral inequalities involving strongly convex functions. J. Funct. Spaces 2018, Article ID 6595921 (2018)

77. Zaheer Ullah, S., Adil Khan, M., Chu, Y.-M.: Majorization theorems for strongly convex functions. J. Inequal. Appl. 2019, Article ID 58 (2019) 
78. Zaheer Ullah, S., Adil Khan, M., Khan, Z.A., Chu, Y.-M.: Integral majorization type inequalities for the functions in the sense of strong convexity. J. Funct. Spaces 2019, Article ID 9487823 (2019)

79. Adil Khan, M., Zaheer Ullah, S., Chu, Y.-M.: The concept of coordinate strongly convex functions and related inequalities. Rev. R. Acad. Cienc. Exactas Fís. Nat., Ser. A Mat. 113(3), 2235-2251 (2019)

80. Chu, Y.-M., Adil Khan, M., Ali, T., Dragomir, S.S.: Inequalities for $\alpha$-fractional differentiable functions. J. Inequal. Appl. 2017, Article ID 93 (2017)

81. Adil Khan, M., Begum, S., Khurshid, Y., Chu, Y.-M.: Ostrowski type inequalities involving conformable fractional integrals. J. Inequal. Appl. 2018, Article ID 70 (2018)

82. Adil Khan, M., Chu, Y.-M., Kashuri, A., Liko, R., Ali, G.: Conformable fractional integrals versions of Hermite-Hadamard inequalities and their generalizations. J. Funct. Spaces 2018, Article ID 6928130 (2018)

83. Adil Khan, M., Iqbal, A., Suleman, M., Chu, Y.-M.: Hermite-Hadamard type inequalities for fractional integrals via Green's function. J. Inequal. Appl. 2018, Article ID 161 (2018)

84. Adil Khan, M., Khurshid, Y., Du, T.-S., Chu, Y.-M.: Generalization of Hermite-Hadamard type inequalities via conformable fractional integrals. J. Funct. Spaces 2018, Article ID 5357463 (2018)

85. Adik Khan, M., Wu, S.-H., Ullah, H., Chu, Y.-M.: Discrete majorization type inequalities for convex functions on rectangles. J. Inequal. Appl. 2019, Article ID 16 (2019)

86. Dragomir, S.S.: On the Hadamard's inequality for convex functions on the co-ordinates in a rectangle from the plane. Taiwan. J. Math. 5(4), 775-788 (2001)

87. Delavar, M.R., Dragomir, S.S.: On $\eta$-convexity. Math. Inequal. Appl. 20(1), 203-216 (2017)

88. Eshaghi Gordji, M., Rostamian Delavar, M., Dragomir, S.S.: Some inequalities related to $\eta$-convex functions. Available at http://www.ajmaa.org/RGMIA/papers/v18/v18a08.pdf

\section{Submit your manuscript to a SpringerOpen ${ }^{\circ}$ journal and benefit from:}

- Convenient online submission

- Rigorous peer review

- Open access: articles freely available online

- High visibility within the field

- Retaining the copyright to your article

Submit your next manuscript at $\gg$ springeropen.com 